\title{
Implications of adopting the WHO 2006 Child Growth Standard in the UK: two prospective cohort studies
}

\author{
C Wright, ${ }^{1}$ R Lakshman, ${ }^{2}$ P Emmett, ${ }^{3}$ K K Ong ${ }^{2,4}$
}

\begin{abstract}
See Perspective, p 549
${ }^{1}$ Division of Developmental Medicine, University of Glasgow, Glasgow, UK; ${ }^{2} \mathrm{MRC}$ Epidemiology Unit, Cambridge, UK; ${ }^{3}$ Community based Medicine, University of Bristol, Bristol, UK; ${ }^{4}$ Department of Paediatrics, University of Cambridge, Cambridge, UK

Correspondence to:

Dr K K Ong, MRC Group Leader \& Paediatric Endocrinologist, MRC Epidemiology Unit, Institute of Metabolic Science, Addenbrooke's Hospital, Box 285, Cambridge CB2 000, UK ken.ong@mrc-epid.cam.ac.uk
\end{abstract}

Accepted 16 September 2007 Published Online First

1 October 2007

\begin{abstract}
Background: The WHO 2006 Child Growth Standard is based on data from international optimally nourished breastfed infants from birth to age 5 years.

Objective: To assess the potential effect of its use on weight and growth monitoring of UK children.

Participants: Full-term members of two populationbased UK birth cohorts: the Children in Focus sub-cohort of the Avon Longitudinal Study of Parents and Children (ALSPAC) ( $n=1335)$ and the Gateshead Millennium Baby Study (GMS; $n=923$ ).
\end{abstract}

Design: Growth data from birth to 5 years were converted into z-scores relative to the WHO 2006 standard.

Results: Compared with the WHO standard, both UK cohorts had higher birth weights (mean z-scores: GMS, 0.17; ALSPAC, 0.34) and ALSPAC had higher birth lengths. After birth, length showed a good fit at all ages. By 2-4 months, both cohorts were similar in weight to the WHO median (mean WHO weight z-score at 4 months: GMS, 0.01; ALSPAC, -0.07), but thereafter the UK cohorts were heavier (mean WHO weight z-score at 12 months: GMS, 0.57; ALSPAC, 0.65). At age 12 months, the risk of being classified as underweight (weight $<2$ nd centile) was considerably lower according to the WHO standard than by the UK 1990 Growth Reference ( $\mathrm{RR}=0.15,95 \% \mathrm{Cl}=0.07$ to 0.32 ), and the risk of being classified as obese at $4-5$ years (body mass index $>98$ th centile) was slightly increased $(\mathrm{RR}=1.35$, $95 \% \mathrm{Cl}=1.02$ to 1.78 ).

Conclusions: Adoption of the WHO 2006 Growth Charts would set a markedly lower standard of weight gain beyond the age of 4 months for UK infants and could support efforts to avoid future childhood obesity. However, the WHO standard is not representative of size at birth in the UK.

The World Health Organisation (WHO) Child Growth Standard for infants and children up to the age of 5 years was published in April 2006. It is based on the growth of healthy breastfed children in optimal conditions between 1997 and 2003 from six different countries: Brazil, Ghana, India, Norway, Oman and USA. ${ }^{2}$ The WHO Multicentre Growth Reference Study (MGRS) collected data for $\sim 8500$ children who were exclusively breast fed for the first 4 months and were living in a well-supported health environment. In consequence, the WHO aims to provide for the first time a standard on "how children should grow", rather than a traditional growth reference that describes "how children are growing".

There is an understandable enthusiasm for the idea of adopting these charts in the UK, but before doing so it is important to assess how well UK children match, or diverge from, the new charts, in order to understand the implications for growth monitoring and clinical care. We have explored this question using data from two representative UK birth cohorts.

\section{METHODS}

The two datasets used were from the Gateshead Millennium Baby Study (GMS) and the Children in Focus sub-sample of the Avon Longitudinal Study of Parents and Children (ALSPAC), which between them provide detailed growth data spanning the entire period of the new charts. GMS is a prospective population-based cohort study of feeding and growth in infancy comprising 1029 babies born between June 1999 and May 2000 in Gateshead, an urban borough in the North of England. For this analysis, data from 923 full-term infants were used. ${ }^{3}$ Birth weight was retrieved from the maternity record, and weights at 12 days, 6-8 weeks, 4 months and 12 months were obtained from the Personal Child Health Records as well as height and weight at school entry. ${ }^{3}$ Half were breast fed at birth, but only $10 \%$ continued breast feeding beyond 4 months.

The ALSPAC Children in Focus sub-cohort includes 1335 full-term infants born in Avon, south-west England, between June and December 1992. Weight and length/height measurements were collected at research clinics at birth, 4 months, 8 months, 12 months, 18 months, 24 months and 5 years. ${ }^{4}$ Just less than half (46\%) were breast fed at age four months (including up to one formula feed per day).

For each child, age- and sex-adjusted z-scores for weight, length (height at $>2$ years old) and body mass index (BMI) were calculated using exact ages at measurement by comparison with both the WHO 2006 and the UK 1990 growth data using software provided respectively by the WHO and the Child Growth Foundation (London, UK). Conditional weight gain was calculated to account for regression to the mean. ${ }^{3}$ Poor infant weight gain was defined as a change in weight SD score $<-1.33 \mathrm{SD}$, which is equivalent to downward crossing through two major centile lines on each growth chart.

Both studies received appropriate ethics committee approval and obtained informed written consent from each participant.

\section{RESULTS}

\section{Comparisons with UK 1990}

Both cohorts showed a reasonably good fit with the UK 1990 reference during the first year of life, 


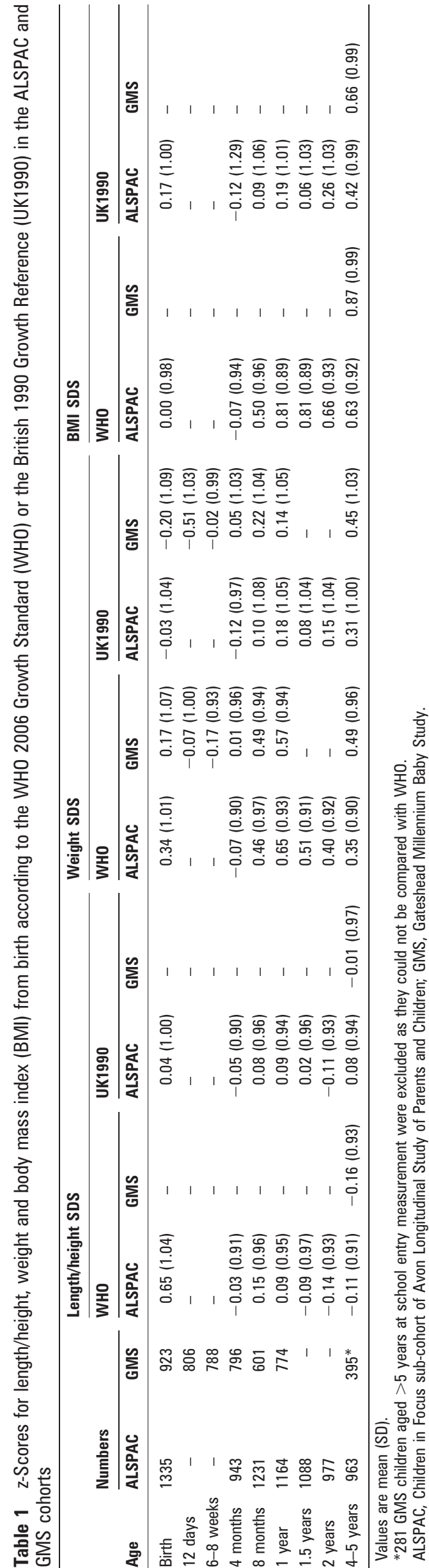

as indicated by mean weight and length $z$-scores close to zero (table 1 and fig 1). The only exception was a transient decline in weight z-score in GMS at age 12 days, which may be expected, as the UK 1990 reference makes no allowance for the physiological neonatal weight loss. By age 4-5 years, weight and BMI z-scores in both cohorts were higher than the UK 1990 average.

\section{Comparisons with WHO 2006}

UK children had relatively high mean z-scores for birth weight and birth length compared with the WHO 2006 standard (table 1). After birth, z-scores for weight in the GMS children rapidly declined towards the WHO median by age 2 weeks, and in both cohorts weight showed a good fit up to 4 months (fig 1). Length and height in both cohorts showed a good fit at all ages after birth (table 1).

Between 4 months and 1 year, compared with the WHO standard, both cohorts showed a rapid rise in mean weight $z-$ scores. After 1 year, the mean $z$-scores as assessed by the different growth reference data started to converge (table 1 , fig 1).

By the WHO 2006 standard, infants were considerably less likely to be classified as underweight (weight $<2$ nd centile; relative risk at 1 year $=0.15 ; 95 \% \mathrm{CI}=0.07$ to 0.32 ) or having poor weight gain (downward-crossing through weight centiles) over the first year, compared with the UK 1990 Reference (table 2). Conversely the proportion of children classified as obese (BMI >98th centile) at age 4-5 years was slightly higher according to the WHO 2006 standard (relative risk $=1.35 ; 95 \%$ $\mathrm{CI}=1.02$ to 1.78 ; table 2 ).

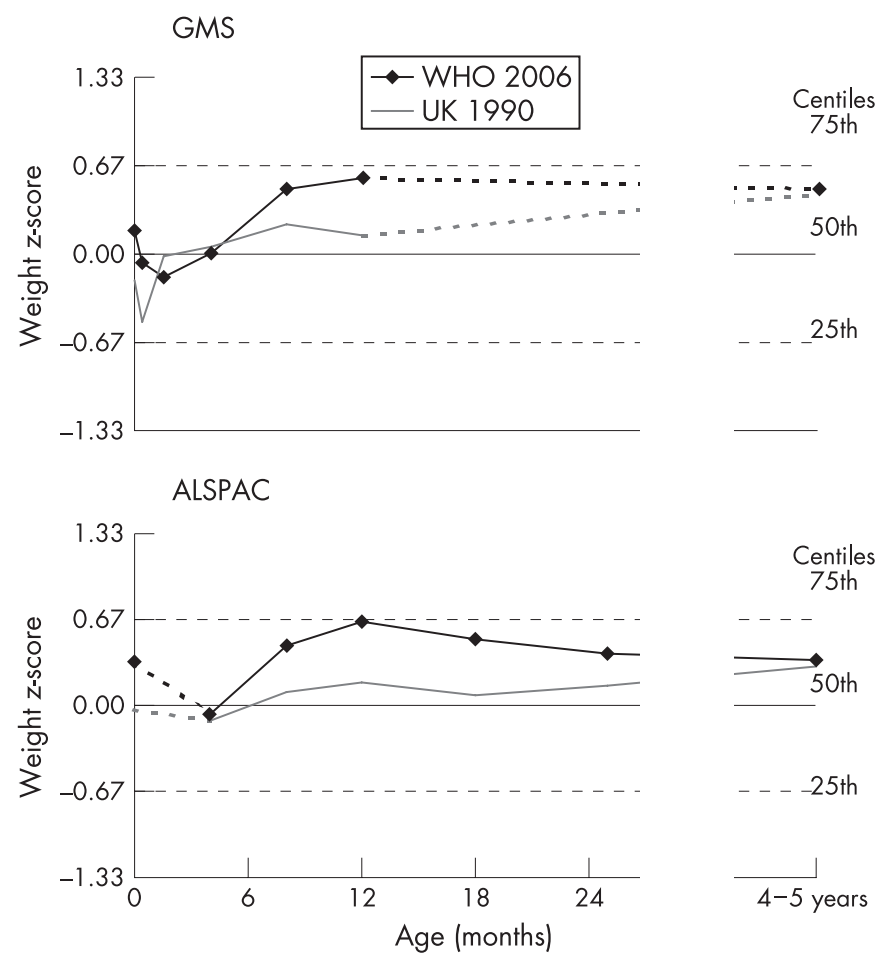

Figure 1 Mean z-scores for weight from birth to 24 months and at 4-5 years, according to WHO 2006 Growth Standard (WHO 2006) or the British 1990 Growth Reference (UK 1990) for the Gateshead Millennium Baby Study (GMS) and the Children in Focus sub-cohort of the Avon Longitudinal Study of Parents and Children (ALSPAC). Dotted lines in each panel indicate the time periods with less density of measurements. 


\section{DISCUSSION}

In summary, adoption of the new WHO growth charts for UK children up to age 5 years would have a significant impact on the interpretation of their weight gain and growth. However, the effects are complex and appear to differ at various ages. The marked reduction in numbers of infants who would be classified as underweight or growth faltering beyond age 4 months is an expected consequence of the WHO's decision to have the breastfed child as the normative model. However, UK infants would also be classified as being larger at birth, but not at 24 months, and would result in a complex pattern of weight centile changes over the first year for the average UK child (fig 1).

This analysis is based on data from two large representative UK birth cohorts, which between them allow comparison with the WHO charts at a wide range of ages. GMS provides detailed weight data early in infancy, and the ALSPAC provides both weight and height/length from infancy through to the preschool years. At times of overlap, the two cohorts showed very close similarity in weights and heights, and, at least in infancy, they are also broadly similar to the UK 1990 reference. The gradual increase in weight $z$-scores by $4-5$ years of age compared with the UK 1990 has been previously reported in ALSPAC and probably reflects the secular changes in UK children. ${ }^{5}$ We are therefore confident that our findings in these two cohorts may be extrapolated to contemporary UK children.

The WHO 2006 Child Growth Standard embodies a number of novel and admirable principles, with the aim of promoting optimal infant and childhood growth. Firstly, the international MGRS source data indicated for the first time that population differences in growth are avoidable, given optimum nutrition and living conditions. ${ }^{6}$ Secondly, the WHO has clearly placed the breastfed child as the norm for growth and development. Conditions of inclusion in the longitudinal component of the MGRS analysis were exclusive or predominant breast feeding up to age 4 months and partial breast feeding to at least 12 months. In consequence, the WHO feels able to publish a standard for optimal growth, rather than simply a description of

Table 2 Percentages of children classified as underweight, poor infant weight gain, or obese according to the WHO Growth Standard (WHO) and the British 1990 Growth Reference (UK1990)

\begin{tabular}{|c|c|c|c|c|c|}
\hline & \multicolumn{2}{|c|}{ ALSPAC } & \multicolumn{2}{|l|}{ GMS } & \multirow{2}{*}{$\begin{array}{l}\text { Combined: } \\
\text { RR (95\% CI) }\end{array}$} \\
\hline & WHO & UK1990 & WHO & UK1990 & \\
\hline \multicolumn{6}{|l|}{ Underweight } \\
\hline 6-8 weeks & - & - & 3.6 & 2.9 & $1.12(0.87$ to 1.43$)$ \\
\hline 4 months & 2.0 & 2.4 & 2.1 & 2.4 & $0.86(0.55$ to 1.33$)$ \\
\hline 8 months & 0.7 & 2.6 & 0.7 & 1.8 & $0.30(0.17$ to 0.56$)$ \\
\hline 1 year & 0.3 & 2.4 & 0.4 & 2.5 & $0.15(0.07$ to 0.32$)$ \\
\hline 1.5 years & 0.6 & 2.9 & - & - & $0.22(0.10$ to 0.48$)$ \\
\hline \multicolumn{6}{|l|}{ Poor infant weight gain } \\
\hline Birth to 1 year & 1.7 & 7.1 & 1.6 & 5.4 & $0.24(0.16$ to 0.36$)$ \\
\hline $6-8$ weeks to 1 year & - & - & 0.4 & 5.4 & $0.08(0.03$ to 0.24$)$ \\
\hline \multicolumn{6}{|l|}{ Obese (\%) } \\
\hline 1 year & 8.7 & 2.7 & - & - & 3.26 (2.21 to 4.83$)$ \\
\hline 1.5 years & 8.0 & 2.6 & - & - & $3.11(2.06$ to 4.71$)$ \\
\hline 2 years & 7.5 & 4.3 & - & - & $1.74(1.20$ to 2.51$)$ \\
\hline $4-5$ years & 7.2 & 5.0 & 10.1 & 8.4 & 1.35 (1.02 to 1.78$)$ \\
\hline
\end{tabular}

ALSPAC, Children in Focus sub-cohort of Avon Longitudinal Study of Parents and Children; GMS, Gateshead Millennium Baby Study; RR, relative risk for each outcome using the WHO standard, compared with the UK 1990 reference; Underweight, weight $<2$ nd centile; Poor infant weight gain, conditional weight gain $<-1.33 \mathrm{SD}$, equivalent to downward crossing through two major centile lines on each growth chart; Obese, body mass index $>98$ th centile.

\section{What is already known on this topic}

- The WHO published new growth charts in April 2006 based on infants of non-smoking, breastfeeding mothers living in optimal conditions in six countries.

- The WHO proposed that these set a standard for normal growth in infancy applicable throughout the world.

\section{What this study adds}

- At birth, UK children are longer and heavier than the WHO standard,

- After birth, the length of UK children matches the WHO standard closely.

- Use of the WHO standard would lead to far fewer UK children being classified as underweight or weight faltering in the first year, but more would be classified as overweight in the preschool years.

- The WHO 2006 Growth Charts would set a lower standard of weight gain for UK infants.

current prevailing growth norms (a "reference"), which may not reflect ideal growth patterns.

However, our findings, particularly during the first 2 months, suggest that these standards may not be simply transferable to the UK. On the WHO chart, UK infants would appear larger than average at birth and then cross approximately half a centile space downwards in the first few weeks of life. The explanation for this may be that, although postnatal nutrition in the WHO MGRS cohort was optimal, intrauterine growth appeared to have been constrained, as size at birth was generally smaller than in the UK. In the MGRS constituent datasets, whereas mean birth weights in Norway and USA $(3.5-3.6 \mathrm{~kg})$ were similar to that in the UK, the populations from several other countries showed markedly lower mean birth weights (3.1 kg in India, and $3.2 \mathrm{~kg}$ in Oman), and this appears to correlate with differences in maternal size. ${ }^{7}$

The UK 1990 and other existing national growth charts do not allow for the rapid weight loss and recovery that normally occurs in the first 2 weeks of life. ${ }^{8}$ This is reflected in fig 1 by a transient dip in the GMS cohort UK 1990 weight SD scores at age 12 days, which probably corrected itself well before their next measurement at age 6 weeks. In contrast, the WHO standard does allow for normal neonatal weight loss. ${ }^{2}$ Therefore, the apparent downward shift in weight centile of UK children on the WHO chart after birth (fig 1) is not simply a transient physiological weight loss, but rather suggests that individual babies with low birth weight in the international MGRS birth cohort showed rapid catch-up growth after birth, even within the first 2 weeks.

Beyond the first 2-4 months, use of the WHO standard would make it much less likely for UK children to be classified as underweight or growth faltering. Recent work has revealed that mild degrees of weight faltering are unlikely to be associated with major social or medical disorders, ${ }^{3}$ and concerns have been expressed that unnecessary parental anxiety may be caused by over-diagnosis. ${ }^{9}$ A change to a new standard, with a more stringent and thus more specific lower threshold, may therefore be timely. 
In contrast with underweight, adoption of the WHO growth chart would make UK infants and toddlers more likely to be classified as overweight or obese. There is a growing body of evidence that a higher plane of growth during infancy is associated with increased risk of obesity in children and adults. ${ }^{11}{ }^{11}$ Although it is not at all clear whether intervention in infancy can have a useful impact on later obesity, presenting the model of slower weight gain during later infancy prescribed by the WHO standard may be beneficial to the long-term health of these children.

The birth weight section of the WHO chart presents other difficulties, as there is no preterm element, which is a well-used feature of UK charts. These two issues taken together suggest that it may not be desirable for the UK to adopt the birth weight section of the WHO chart, beginning its use instead after the first 2 weeks.

In conclusion, the WHO 2006 Growth Standard places the breastfed child as the norm for growth. Its use would greatly reduce the numbers of UK infants classified as underweight and support efforts to avoid excess weight gain in infancy. However, the WHO 2006 Growth Standard is not representative of size at birth in the UK. In view of the resulting complex weight centile changes in the first few weeks of life, the potential confusion about feeding that this might raise with mothers, and also the absence of a preterm element to the WHO charts, the Department of Health Scientific Advisory Committee on Nutrition and the Royal College of Paediatrics and Child Health have recently jointly recommended that the WHO 2006 Growth Standard is appropriate for use in the UK children, but only from age 2 weeks. ${ }^{12}$ For birth weight, the UK 1990 reference would continue. The consequences of these recommendations for monitoring of infant weight gain in the UK are likely to be widespread and will need careful and coordinated consideration.
Acknowledgements: KKO and CMW are members of a Joint Expert Group of the Scientific Advisory Committee on Nutrition and the Royal College of Paediatrics and Child Health tasked with considering the implications of the WHO Growth Standards in the UK. Many of the current data were presented to that Expert Group and have been released for consultation. The views expressed in this article are specific to the authors.

We are extremely grateful to all the families who took part in the ALSPAC and GMS studies and their research teams.

Funding: UK Medical Research Council, the Wellcome Trust and the University of Bristol provide core provide support for ALSPAC. The infancy phase of the GMS was funded by the Henry Smith Charity, SPARKS, Northern and Yorkshire NHS R\&D and Gateshead NHS R\&D.

Competing interests: None.

\section{REFERENCES}

1. de Onis M, Garza C, Victora CG, et al. The WHO Multicentre Growth Reference Study: planning, study design, and methodology. Food Nutr Bull 2004;25:S15-26.

2. WHO Child Growth Standards based on length/height, weight and age. Acta Paediatr Supp/ 2006:450:76-85.

3. Wright CM, Parkinson KN, Drewett RF. The influence of maternal socioeconomic and emotional factors on infant weight gain and weight faltering (failure to thrive): data from a prospective birth cohort. Arch Dis Child 2006;91:312-17.

4. Ong KK, Ahmed ML, Emmett PM, et al. Association between postnatal catch-up growth and obesity in childhood: prospective cohort study. $B M$ 2000;320:967-71.

5. Reilly J, Dorosty A, Emmett P. Prevalence of overweight and obesity in British children: cohort study. BMJ 1999;319:1039.

6. Assessment of differences in linear growth among populations in the WHO Multicentre Growth Reference Study. Acta Paediatr Supp/ 2006;450:56-65.

7. Enrolment and baseline characteristics in the WHO Multicentre Growth Reference Study. Acta Paediatr Supp/ 2006;450:7-15.

8. Wright CM, Parkinson KN. Postnatal weight loss in term infants: what is normal and do growth charts allow for it? Arch Dis Child Fetal Neonatal Ed 2004;89:F254-7.

9. Spencer NJ. Failure to think about failure to thrive. Arch Dis Child 2007:92:95-6.

10. Baird J, Fisher D, Lucas $P$, et al. Being big or growing fast: systematic review of size and growth in infancy and later obesity. BMJ 2005:331:929.

11. Ong KK, Loos RJ. Rapid infancy weight gain and subsequent obesity: systematic reviews and hopeful suggestions. Acta Paediatr 2006:95:904-8.

12. Department of Health. Application of the WHO Growth Standards in the UK. http:// www.sacn.gov.uk/ (accessed 21 Nov 2007)

\section{Save your favourite articles and useful searches}

Use the "My folders" feature to save and organise articles you want to return to quickly-saving space on your hard drive. You can also save searches, which will save you time. You will only need to register once for this service, which can be used for this journal or all BMJ Journals, including the BMJ. 